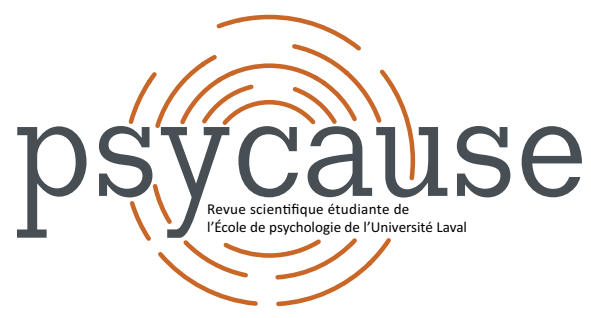

\title{
PSYCAUSE
}

Revue scientifique étudiante de l'École de psychologie de l'Université Laval

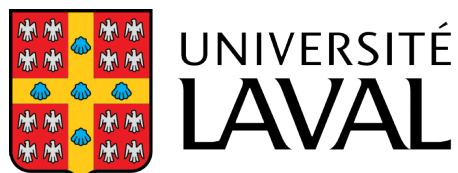

Faculté des sciences sociales École de psychologie

NOVEMBRE 2020 - VOL. $10 \mathrm{~N}^{\circ} 2$

\section{ÉVOLUTION ET FACTEURS ASSOCIÉS AU BIEN-ÊTRE DES INDIVIDUS AYANT UN TRAUMATISME CRANIO-CÉRÉBRAL À L'ÂGE ADULTE}

Olivier AUBUCHON${ }^{1}$, Jessica HORTH ${ }^{1}$, Meili LANTEIGNE \& Marie-Christine OUELLET ${ }^{1}$

${ }^{1}$ École de psychologie, Université Laval

*olivier.aubuchon.1@ulaval.ca

\section{Pour citer l'article}

Aubuchon, O., Horth, J., Lanteigne, M., \& Ouellet, M.-C. (2020). Évolution et facteurs associés au bien-être des individus ayant un traumatisme cranio-cérébral à l'âge adulte. Psycause: Revue scientifique étudiante de l'École de psychologie de I'Université Laval, 10(2), 9-11. 


\title{
ÉVOLUTION ET FACTEURS ASSOCIÉS AU BIEN-ÊTRE DES INDIVIDUS AYANT UN TRAUMATISME CRANIO-CÉRÉBRAL À L'ÂGE ADULTE
}

\author{
Olivier AUBUCHON ${ }^{1}$, Jessica HORTH ${ }^{1}$, Meili LANTEIGNE ${ }^{1}$ \& Marie-Christine OUELLET ${ }^{1}$ \\ ${ }^{1}$ École de psychologie, Université Laval \\ *olivier.aubuchon.1@ulaval.ca
}

Mots-clés : Bien-être, traumatisme cranio-cérébral, participation sociale, stratégies d'adaptation, soutien social

Un traumatisme cranio-cérébral (TCC) peut engendrer plusieurs séquelles chroniques sur la santé, dont des limites fonctionnelles pouvant affecter le retour au travail (Gollaher et coll., 1998). Alway, Gould, Johnston, McKenzie, et Ponsford (2016) démontrent que $75,2 \%$ des personnes de leur échantillon ( $N=161$ ) ayant un TCC modéré ou grave présente au moins un trouble psychologique dans les cinq premières années post-TCC, soulignant le risque pour la santé mentale. Bien que les difficultés associées au TCC soient fréquemment soulevées, peu d'études abordent le bien-être. Quelques études démontrent que le soutien social (Holland et Schmidt, 2015), la participation sociale (Vos, Poritz, Ngan, Leon-Novelo, \& Sherer, 2019) et I'utilisation de stratégies d'adaptation axées sur les problèmes (Hanks et coll., 2014) sont associés à moins de symptômes psychologiques post-TCC. Une meilleure compréhension de l'évolution et des facteurs associés au bien-être postTCC est pertinente pour favoriser une meilleure adaptation. Aucun consensus n'existe sur la définition du bien-être après un TCC. Conséquemment, cette étude adopte une vision large en englobant les principaux impacts d'un TCC, soit sur la santé mentale, le retour à une vie active, la cognition et la douleur. Les objectifs de cette étude sont de (1) documenter l'évolution du nombre d'individus présentant un bien-être élevé dans les trois premières années post-TCC, (2) comparer un groupe de bien-être élevé à un groupe de bien-être moindre sur des caractéristiques sociodémographiques et cliniques, et (3) d'identifier des facteurs psychosociaux à 8 mois qui prédisent l'appartenance au groupe de bien-être élevé à 36 mois.

\section{Méthode}

Cent quatre-vingt-un adultes ayant un TCC ontété rencontrés à $4,8,12,24$ et 36 mois post-TCC pour une entrevue semi-structurée et la complétion de questionnaires. L'échantillon a été divisé en deux groupes (bien-être élevé et bien-être moindre). Pour être dans le groupe de bienêtre élevé, les individus devaient présenter quatre critères de bien-être: peu de douleur (score supérieur à 30 à la sous-échelle de douleur du Medical Outcome Study: Short Form Health Survey, SF-36), peu de plaintes cognitives (score supérieur à 12 à la sous-échelle cognitive du Medical Outcomes Study, MOS-COG), une absence de troubles psychologiques au Structured Clinical Interview for DSM-IV (SCID-IV) et un retour à une vie active (bénévolat, études ou travail), à temps plein ou partiel, aux questionnaires sociodémographiques. Les individus du groupe de bien-être moindre présentaient de la douleur, des plaintes cognitives, un trouble psychologique et/ou l'absence d'une vie active.

Des prédicteurs potentiels du bien-être post-TCC ont été mesurés à 8 mois, soit: (1) le score de participation sociale au Participation Assessment with Recombined ToolsObjective (PART-O), en utilisant les sous-échelles Relations sociales et Allées et venues, mais en excluant la souséchelle de Productivité, mesurée pour le retour à une vie active, (2) les scores de trois stratégies d'adaptation au Brief Coping Orientation to Problem Experiences (COPE), axées sur les problèmes (p.ex. recherche active de solutions), les émotions (p.ex. recherche de support émotionnel) et les stratégies passives émotionnelles (p.ex. consommation de substances), et (3) le score de soutien social au Modified Medical Outcomes Study Social Support Survey (mMOS-SS).

\section{Ānalyses et résultats}

Des proportions ont été calculées à chaque temps de mesure afin d'identifier le pourcentage de l'échantillon correspondant au groupe de bien-être élevé (objectif 1). Une analyse visuelle indique une augmentation des individus inclus dans le groupe de bien-être élevé entre 4 et 36 mois post-TCC (Figure 1).

Les deux groupes ont été comparés sur des données sociodémographiques (sexe, statut marital, âge, années de scolarité) et cliniques (historique de troubles psychologiques, sévérité du TCC, échelle de Glasgow, nombre de blessures orthopédiques, durée d'hospitalisation) à 36 mois par des analyses de khi-carré et de test-t (objectif 2). Les analyses ont été effectuées sur 103 participants détenant des données complètes. Les résultats démontrent que seul I'historique de troubles psychologiques pré-TCC est significativement moins présent dans le groupe de bien-être élevé $\left(X^{2}(1, N=\right.$ 103) $=5,13, p=0,024$ ).

Une analyse de régression logistique a été effectuée auprès de 97 participants détenant des données complètes afin d'identifier des variables prédisant l'appartenance au groupe 


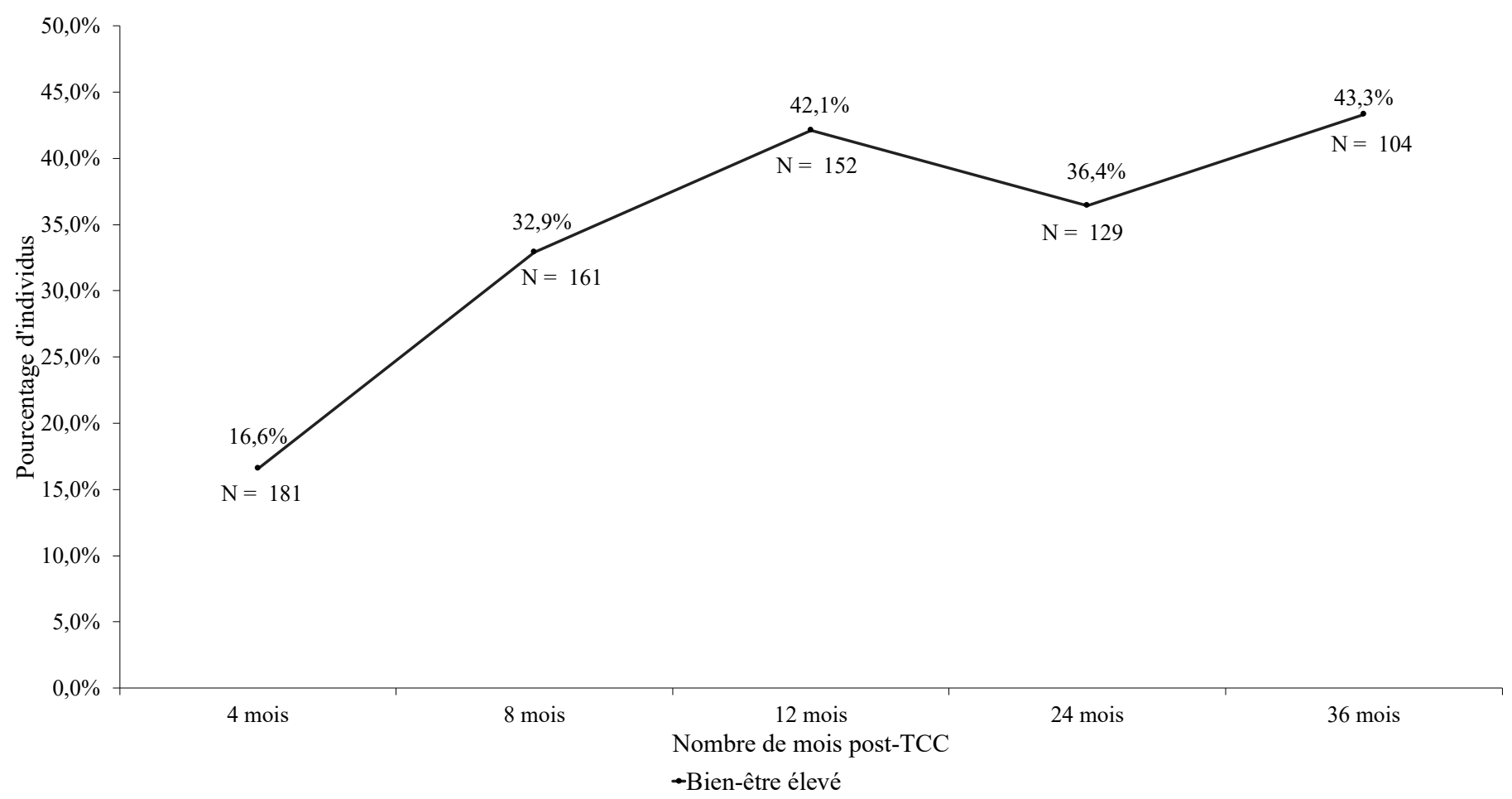

Figure 1. Proportion des individus dans le groupe de bien-être élevé entre 4 et 36 mois post-TCC.

de bien-être élevé (objectif 3). Étant donné leur corrélation significative $(0,60)$, les échelles des stratégies axées sur les problèmes et sur les émotions du COPE ont été fusionnées. Les résultats indiquent, avec l'historique de troubles psychologiques pré-TCC, qu'un score de participation sociale plus élevé au PART-O à 8 mois prédit l'appartenance au groupe de bien-être élevé à 36 mois $(B=1,13, E . S=$ 0,44 , Wald $=6,50, p=0,011$ ) (Tableau 1).

\section{Discussion}

Les résultats suggèrent que dans les trois premières années post-TCC, la proportion d'individus ayant un niveau plus élevé de bien-être augmente. De plus, le fait d'avoir souffert d'une problématique psychologique pré-TCC affecte le bien-être, d'où l'importance de bien évaluer le passé psychiatrique. Finalement, les résultats suggèrent également qu'intervenir sur la participation sociale dans la première année post-TCC pourrait améliorer le bien-être à long terme. Les futures études pourraient investiguer l'effet sur le bien-être de différentes interventions favorisant la participation sociale (loisirs, activité physique, bénévolat) et le soutien social.

\section{Références}

Alway, Y., Gould, K. R., Johnston, L., McKenzie, D. et Ponsford, J. (2016). A prospective examination of Axis I psychiatric disorders in the first 5 years following moderate to severe traumatic brain injury. Psychological Medicine, 46(6), 1331 1341. http://doi.org/10.1017/S0033291715002986

Gollaher, K., High, W., Sherer, M., Bergloff, P., Boake, C., Young, M. E. et Ivanhoe, C. (1998). Prediction of employment outcome one to three years following traumatic brain injury (TBI). Brain Injury, 12(4), 255-263. http://doi. org/10.1080/026990598122557

Tableau 1

Résultats de l'analyse de régression logistique des prédicteurs (à 8 mois) du bien-être à 36 mois.

\begin{tabular}{lcccc}
\hline Prédicteurs & $B$ & Wald & $p$ & Rapport de cotes \\
\hline Participation sociale & 1,132 & 6,500 & $0,011^{*}$ & 3,101 \\
Soutien social & $-0,005$ & 0,175 & 0,675 & 0,995 \\
Stratégies d'adaptation émotionnelles passives & $-0,191$ & 0,657 & 0,417 & 0,826 \\
Stratégies d'adaptation axées sur les problèmes et sur les émotions & 0,297 & 1,255 & 0,263 & 1,345 \\
Historique de troubles psychologiques & $-0,623$ & 1,778 & 0,182 & 0,536 \\
\hline
\end{tabular}

Note. $N=97 ;{ }^{\star} p<0,05$ 
Hanks, R. A., Rapport, L. J., Waldron-Perrine, B. et Millis, S. R. (2014). Role of character strengths in outcome after mild complicated to severe traumatic brain injury: a positive psychology study. Archive of Physical Medicine and Rehabilitation, 95(11), 2096-2102. http://doi.org/10.1016/j. apmr.2014.06.017

Holland, J. N. et Schmidt, A. T. (2015). Static and dynamic factors promoting resilience following traumatic brain injury: A brief review. Neural Plasticity, 2015, 1-8. http:// doi.org/10.1155/2015/902802

Vos, L., Poritz, J. M. P., Ngan, E., Leon-Novelo, L. et Sherer, M. (2019). The relationship between resilience, emotional distress, and community participation outcomes following traumatic brain injury. Brain Injury, 1-9. http://doi.org/10.10 80/02699052.2019.1658132wise

\title{
Pour citer l'article
}

Aubuchon, O., Horth, J., Lanteigne, M., \& Ouellet, M.-C. (2020). Évolution et facteurs associés au bien-être des individus ayant un traumatisme cranio-cérébral à l'âge adulte. Psycause: Revue scientifique étudiante de l'École de psychologie de I'Université Laval, 10(2), 9-11.

\section{IMPULSIVITÉ ET DISTRACTIBILITÉ : LES CONVERSATIONS TÉLÉPHONIQUES EN ARRIĖRE-PLAN SONT-ELLES PARTICULIÈREMENT DÉRANGEANTES?}

\author{
Claudia BABIN ${ }^{1 * \forall}$, Carole-Anne BOULET ${ }^{1 \forall}$, Alex PÉPIN ${ }^{1 \forall}$, Charlélie BÉNARD ${ }^{1}$ \& François VACHON ${ }^{1}$ \\ ${ }^{1}$ École de psychologie, Université Laval \\ * claudia.babin.1@ulaval.ca \\ ${ }^{\forall}$ Contribution égale des auteurs et autrices
}

Mots-clés : Impulsivité, distraction, bruit ambiant, bureau à aire ouverte, attention

L'impulsivité est un trait de personnalité qui influence le comportement humain et qui doit être considéré sur un continuum. Étant donné le peu de consensus quant à une définition globale de celle-ci, des chercheurs recommandent d'étudier l'impulsivité en fonction de ses composantes comportementales. Plusieurs modèles théoriques identifient une composante reliée au contrôle inhibiteur, soit la capacité d'un individu à faire fi d'éléments qui sont non pertinents et potentiellement distrayants. Stahl et ses collaborateurs (2014) associent notamment l'impulsivité à des difficultés dans le contrôle de l'interférence causées par un stimulus, de sorte qu'une plus faible résistance à la distraction pourrait se manifester chez les individus plus impulsifs. Ainsi, en raison de cette plus grande susceptibilité à la distraction, ces derniers pourraient être désavantagés dans des contextes caractérisés par une omniprésence de stimulations sonores non pertinentes, comme les bureaux à aire ouverte.

Parmi les diverses sources de bruit ambiant que l'on retrouve dans ce type d'environnement, les conversations en arrièreplan sont particulièrement dérangeantes, et ce, autant entre des personnes que via un appel téléphonique. Cependant, les conversations téléphoniques auraient un pouvoir distracteur plus grand que les conversations avoisinantes, puisqu'un seul des interlocuteurs peut être entendu. Ce discours dans lequel une partie de la conversation demeure toujours inaudible, appelé demilogue, nuirait davantage à la cognition qu'un dialogue, où les deux interlocuteurs sont entendus (Marsh et coll., 2018). La baisse de performance observée en présence d'un demilogue relativement à celle d'un dialogue est appelée effet de demilogue. Elle serait expliquée par la tendance naturelle de l'être humain à vouloir compléter ce qui est incomplet, ainsi que par la capture de l'attention due au besoin d'écouter (Monk, Fellas, \& Ley, 2004). Ce besoin d'écouter et de comprendre la seconde moitié de la conversation se traduit par une écoute non intentionnelle où l'attention est involontairement détournée vers ce discours. Ainsi, si l'impulsivité est réellement liée à une plus grande distractibilité, ce besoin d'écouter engendré par le demilogue devrait rendre les individus plus impulsifs davantage sensibles à l'effet de demilogue. La présente étude vise à vérifier si le trait d'impulsivité influence la vulnérabilité à la distraction causée par ces conversations téléphoniques en arrière-plan. Si tel est le cas, il est attendu que la magnitude de l'effet de demilogue soit plus grande chez les individus du groupe d'impulsivité élevée, comparativement au groupe d'impulsivité faible. 